\title{
Interventional radiology at the meetings of the German Radiological Society from 1998 to 2008: evaluation of structural changes and radiation issues
}

\author{
Stefan P. Lemburg • Daniela Roggenland • \\ Volkmar Nicolas $\cdot$ Christoph M. Heyer
}

Received: 29 June 2011 /Revised: 11 October 2011 / Accepted: 19 October 2011 / Published online: 1 December 2011

(C) European Society of Radiology 2011

\begin{abstract}
Objectives Evaluation of structural changes and the weight given to radiation exposure of interventional radiology (IR) contributions at the Congress of the German Radiological Association from 1998 to 2008.

Methods All IR abstracts were evaluated for type of contribution, design, imaging modality, and anatomic region. Weight given to radiation exposure was recorded as general statement, main topic and/or dose reduction. Statistical analysis included calculation of absolute/relative proportions of subgroups and ANOVA regression analyses. Results Out of 9,436 abstracts, 1,728 (18\%) were IR-related. IR abstracts significantly rose to a maximum of $200(20 \%)$ in 2005 ( $P=0.048)$. While absolute numbers of scientific contributions declined, educational contributions significantly increased $(P=0.003)$. Computed tomography $(\mathrm{CT})$ and magnetic resonance imaging (MRI) were the main IR imaging modalities, with growing use of CT $(P=0.021)$. The main body regions were vessels (45\%) and abdomen (31\%). Radiation exposure was addressed as a general statement in $3 \%$ of abstracts, as a main topic in $2 \%$, and for dose reduction in $1 \%$, respectively. During the study interval a significant growth of dose reduction abstracts was observed $(P=0.016)$.

Conclusions IR emerged as a growing specialty of radiology, with a significant increase in educational contributions. Radiation exposure was rarely in the focus of interest but
\end{abstract}

S. P. Lemburg • D. Roggenland • V. Nicolas $•$ C. M. Heyer $(\triangle)$ Institute of Diagnostic Radiology,

Interventional Radiology and Nuclear Medicine,

Berufsgenossenschaftliches Universitätsklinikum

Bergmannsheil GmbH, Ruhr-University of Bochum,

Buerkle-de-la-Camp Platz 1,

44789 Bochum, Germany

e-mail: christoph.heyer@rub.de contributions relating to dose reduction demonstrated a significant growth during the study period.

Main Messages

- Interventional radiology emerged as a growing specialty at the German radiological congress.

- Significant increments of educational and prospective research contributions could be observed.

- Despite a significant trend towards computed tomography, radiation exposure of IR was rarely in the focus of interest.

- Contributions related to dose reduction demonstrated a significant growth during the study period.

Keywords Interventional radiology Radiation exposure · Dose reduction $\cdot \mathrm{CT} \cdot \mathrm{MRI}$

\section{Introduction}

Image-guided interventions are an acknowledged instrument in the diagnosis and therapy of a broad spectrum of diseases. Beside diagnostic procedures for harvesting histological specimens, Interventional Radiology (IR) covers a vast range of procedures such as dilatation or stenting of vessels and other tubular structures, embolisation of haemorrhages, draining of localised fluid collections, and catheter insertions to different body cavities [1]. In all cases, image guidance has become a necessary prerequisite to localise the target region, guide and document optimal deployment of the interventional device, and to evaluate success, failure or complications of the procedure. In several clinical situations, IR has proven to be a reasonable, safe, and cost-effective alternative to other therapeutic options such as open surgery [2,3]. With a growing proportion of elderly patients with higher comorbidity due to chronic diseases and, hence, fewer options 
for more invasive approaches, an increasing demand for IR experts and procedures is notable [1, 4-8].

Although interventions can principally be performed with all imaging modalities, computed tomography (CT) as a cross-sectional imaging method has several advantages as a guiding instrument. Due to its wide availability, CT imaging today is easy to achieve and available around the clock in most medical facilities. Moreover, CT produces non-superimposed images of anatomically complex body regions, does not interfere with most interventional devices, is increasingly resistant to motion artefacts due to rapid scanning, and is not hindered by bony structures. Thus, CT presents clear advantages for guiding interventions compared with other imaging modalities. One main disadvantage of $\mathrm{CT}$ is the concomitant radiation exposure, a topic that has incrementally evoked discussions about the appropriate use and concomitant risk of ionising imaging examinations in medicine - not only amongst professional health carers but also in the lay press. Being ex officio responsible in most interventional procedures with concomitant radiation exposure, interventional radiologists play the leading role in the appropriate application of ionising imaging modalities and the preservation of radiation protection.

While the occupation with radiation exposure at the congress of the German Radiological Association (DRK) has been investigated with respect to general radiology and paediatric radiology from 1998-2008 elsewhere [9, 10], little is known about the structure and quantity of IR contributions in scientific and educational meetings or congresses pertaining to the topics of the applied imaging modalities (especially with emphasis on CT usage) and the awareness of radiation exposure concomitant with IR procedures.

The aim of the presented study was the systematic evaluation of structural changes of IR contributions and the weight given to radiation exposure related to IR at the DRK in an 11-year period from 1998 to 2008.

\section{Materials and methods}

The study was based on the abstracts of the scientific programs of the DRK congress from 1998 to 2008 [11] as published by Georg Thieme Verlag (Stuttgart/New York) publishers, therefore taking into account the 79th to 89th DRK.

The two underlying assumptions for this investigation were that (1) the DRK accurately reflects the current state of scientific endeavour in German-speaking countries and (2) that the published abstracts contain all pertinent findings. Thus, the summarisations were considered to adequately state information on objectives, methods and results, as well as the authors' conclusions. Moreover, any subject not expressly mentioned in the abstract should in fact not have been primary content of the investigation. Congress contributions that could not be evaluated (withdrawn contributions, missing abstract texts, etc.) were not included in the analysis.

Every available abstract was evaluated according to the following variables. First, the thematic category of the contribution was documented as interventional versus noninterventional abstract. The type of contribution as scientific presentation, scientific poster, educational poster, workshop, refresher course, multimedia, highlight session, inventor's forum, radiology technician educational course and radiological technician clinical seminar was noted. Furthermore, the type of scientific study was assessed as prospective or non-prospective. All abstracts were checked for the imaging modalities used in IR as CT, magnetic resonance imaging (MRI), conventional radiography, angiography, ultrasound, and fluoroscopy. The anatomic region of IR procedure was noted as chest, heart, breasts, abdomen/pelvis, central nervous system including head/ neck, musculoskeletal system, and vascular area (encompassing arterial, venous, or lymphatic vessels).

The weight given to the topic of radiation exposure was recorded in three categories: (1) a general statement on radiation exposure, if radiation dose was addressed in any form (e.g. concrete mention of dose-relevant terms such as "radiation dose", "radiation exposure", "radiation burden", etc. and precise specification of exposure as effective dose reports in $\mathrm{mSv}$, etc.), (2) radiation exposure as main topic, if radiation dose was the primary subject of the contribution, and/or (3) radiation protection, if dose reduction was the primary subject of the contribution. Multiple naming was taken into consideration for body region, imaging modality (e.g. comparison of two imaging modalities in IR procedures) and the three dose categories.

Statistical analysis was conducted with PASW Statistics, version 18.0.0 (SPSS, Chicago, IL, USA), calculating absolute and relative proportions of the investigated subgroups in the context of all contributions to the DRK from 1998 to 2008. ANOVA regression analysis was performed for: absolute numbers of all IR contributions, scientific, non-scientific and prospective IR contributions, contributions related to $\mathrm{CT}$, combined non-CT ionising and non-ionising imaging modalities, and IR contributions relating to the dose categories. Significance level was set to $5 \%$.

\section{Results}

Of 9,472 scientific contributions presented at the DRK during the study period, 9,436 (99.6\%) were eligible for inclusion in our evaluation. The average number of published abstracts per congress per year was 858 , ranging from 705 (2001) to 987 (2005). Abstracts related to IR counted for 1,728 of all abstracts in this 11-year period with a mean value of 157 IR abstracts per year (18.3\%). 
Abstracts dealing with IR rose significantly from a minimum of 135 abstracts $(15.6 \%)$ in 1998 to a maximum of 200 abstracts $(20.3 \%)$ in 2005 ; $P=0.048$ (Fig. 1).

\section{Structure of IR contributions}

IR contributions covered all categories of the DRK program with $1,153 / 1,728(67.9 \%)$ being scientific presentations (mean $n=105 /$ year) and 245/1,728 (14.2\%) scientific posters (mean $n=22 /$ year). Due to considerable changes in the structure of the DRK during the study period, only scientific posters and scientific presentations were noted in all DRK programs of the study period. During the study period, $n=98(7.3 \%)$ workshops (mean $n=12 /$ year), $n=9$ (1.7\%) educational posters (mean $n=3 /$ year), $n=190$ (14.2\%) refresher courses (mean $n=24 /$ year), $n=7$ (1.2\%) multimedia presentations (mean $n=2 /$ year), $n=3(2.2 \%)$ highlight sessions, $n=2(1.3 \%)$ contributions to the inventors' forum, $n=15(1.7 \%)$ radiology technician educational courses (mean $n=3 /$ year), and finally $n=5(0.8 \%)$ radiological technician clinical seminars (mean $n=1 /$ year) were IR-related. Relative proportions of scientific contributions in IR diminished with scientific IR posters being halved in 2008, but absolute numbers of all scientific IR contributions did not decline significantly. Non-scientific IR contributions demonstrated a highly significant increase from 7 to $33 \%$ with a mean value of 30 contributions per year; $P=0.003$ (Fig. 2). Of all IR abstracts, 166 (9.5\%) showed a prospective study design with $20 / 245$ (9.3\%) being scientific posters and 146/1,153 (13.1\%) scientific presentations. Based on a significant growth in absolute numbers $(P=0.038)$, the relative proportion of prospective IR abstracts showed an increase from $4.5 \%$ in 1999 to a maximum of $14.5 \%$ in 2008 . Absolute numbers remained small with a maximum of six prospective scientific posters in 2005 and 21 prospective scientific presentations in 2008.
In relation to the described imaging modalities, the sum and mean percentage of prospective contributions during the whole study period was $23(7.1 \%)$ for CT, $25(7.0 \%)$ for MRI, seven (8.2\%) for ultrasound, and $32(10.5 \%)$ combined for non-CT ionising imaging modalities [with $n$ $=28(10.1 \%)$ for angiography, $n=3(5.3 \%)$ for fluoroscopy, and $n=1(3 \%)$ for conventional radiography; respectively]. Table 1 presents these results.

Imaging modalities and body regions

Over the entire period, cross-sectional imaging modalities were the main imaging modalities of IR with CT encompassing $n=320(18.1 \%)$ and MRI $n=330(19.1 \%)$ of all IR-related abstracts, respectively. CT contributions in IR demonstrated a significant increase of $16.9 \%$ in 1998 to $23.5 \%$ in $2008(P=0.021)$. Non-CT IR imaging modalities with ionising radiation were presented in 223 abstracts $(12.5 \%)$ with 180 abstracts $(10.2 \%)$ relating to angiography, $37(2 \%)$ to fluoroscopy, and $6(0.3 \%)$ to conventional radiography. ionising non-CT IR imaging modalities declined in their proportion from $11.8 \%$ (1998) to $5.4 \%$ (2008) with a peak in 2003-2005 (26.9-32.5\%), mainly attributed to angiography. Combined non-CT, non-ionising imaging modalities (i.e. MRI and ultrasound) did not demonstrate significant numerical changes, with MRI declining from $20.6 \%$ in 1998 to $15.1 \%$ in 2008 and ultrasound displaying stable contributions with a mean value of $4.6 \%$ (Fig. 3). With respect to the body regions, principally presented locations of IR were vessels with $n=760$ (44.9\%) and abdomen with $n=537$ (30.7\%). Breasts, chest including lungs, bones and muscles, and central nervous system including head and neck accounted for 137 (8.1\%), 153 (8.6\%), $172(9.6 \%)$, and $146(8.5 \%)$ abstracts, respectively. The heart region or paediatric interventions were recorded at $\leq 3 \%$. While abstracts concerning vessel interventions dem-
Fig. 1 Absolute number of contributions in IR. All all DRK contributions, $I R$ all IR contributions, SC IR scientific contributions in IR, Non-Sc IR non-scientific contributions in IR

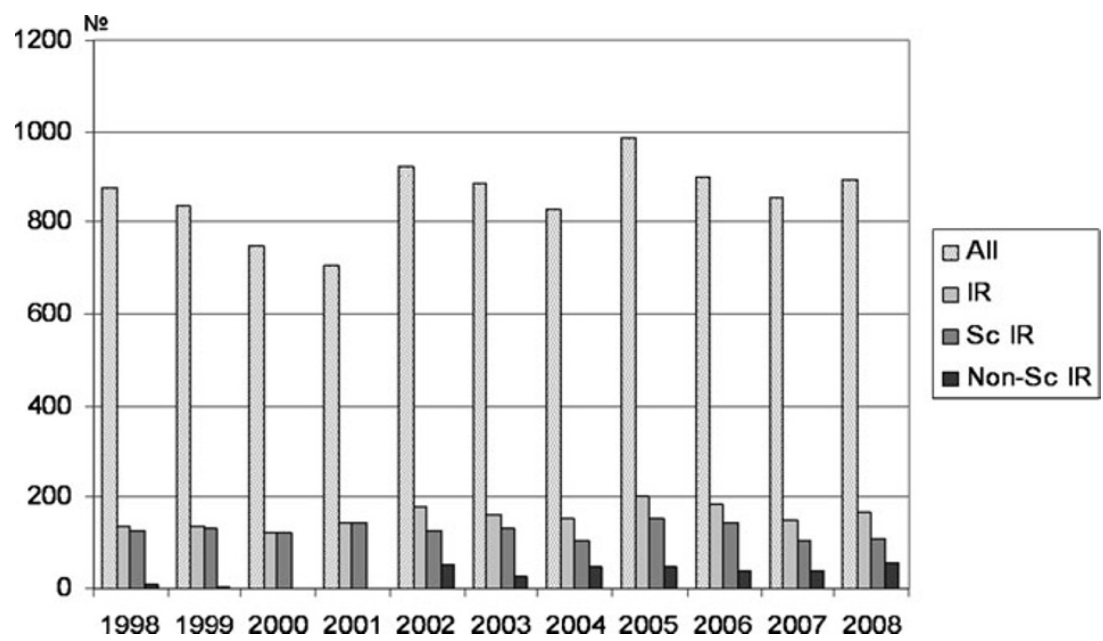


Fig. 2 Relative proportions of IR contributions for scientific poster categories. Tec educational courses for technicians, $W / R$ workshops and refresher courses, Sc Present scientific presentations, $D R K$ Congress of the German Radiological Society, Other multimedia/ highlight session, inventor's forum, etc., Ed Posters educational posters, Sc Posters scientific posters

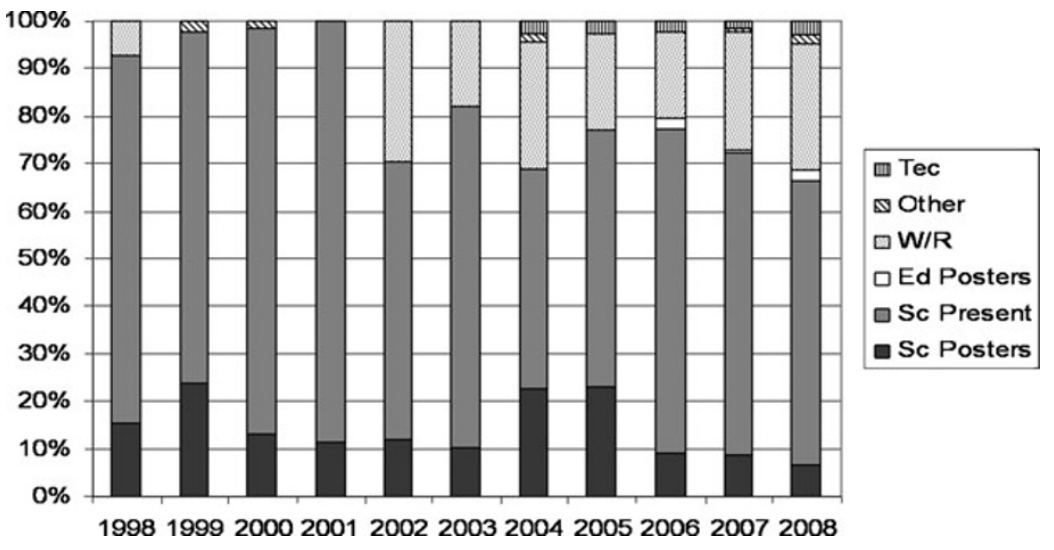

onstrated a drop in percentages, contributions relating to interventions of the abdomen, chest and musculoskeletal system increased in their relative proportions (Fig. 4).

\section{Radiation exposure and IR}

The issue of radiation exposure was addressed over the entire study period as a general statement in 56 IR abstracts (3\%). Thirty-one contributions $(1.6 \%)$ of all dealt with radiation exposure as the main topic and $22(1.3 \%)$ were involved with dose reduction. The relative proportion of dose-related abstracts increased from a minimum of $2.4 \%$ in 2000 to a maximum of $11 \%$ in 2005 . On the basis of small absolute values, a significant growth of contributions relating to dose reduction $(P=0.016)$ could be observed, while the two other dose categories also increased, albeit non-significantly (Fig. 5). Percentages of abstracts dealing with radiation dose demonstrated a general increase for all examined dose categories, in correlation most pronounced in the category of dose reduction (Fig. 6). With respect to $\mathrm{CT}$ and ionising non-CT imaging modalities, abstracts relating to CT occurred in 20 contributions in the sum of general statements on radiation (6.5\% of all CT abstracts), radiation exposure as the main topic occurred in ten abstracts (2.9\% of all CT abstracts), and dose reduction themes occurred in seven contributions $(1.8 \%$ of all CT abstracts). ionising non-CT imaging modalities demonstrated higher values in all three dose categories [20 (9.6\%), 15 $(8.6 \%)$, and $12(7.9 \%)$, respectively], as presented in Table 2.

Table 1 Numbers and percentages of prospective scientific contributions in IR

\begin{tabular}{|c|c|c|c|c|c|c|c|c|c|c|c|c|c|}
\hline \multirow[t]{3}{*}{ Years } & \multirow{3}{*}{$\begin{array}{l}\text { AII IR } \\
n\end{array}$} & \multicolumn{4}{|c|}{ All scientific contributions } & \multicolumn{4}{|c|}{ Scientific posters } & \multicolumn{4}{|c|}{ Scientific presentations } \\
\hline & & \multicolumn{2}{|l|}{ All } & \multicolumn{2}{|c|}{ Prospective } & \multicolumn{2}{|l|}{ All } & \multicolumn{2}{|c|}{ Prospective } & \multirow{2}{*}{$\begin{array}{l}\text { All } \\
n\end{array}$} & \multicolumn{3}{|c|}{ Prospective } \\
\hline & & $n$ & $\%$ & $n$ & $\%$ & $n$ & $\%$ & $n$ & $\%$ & & $\%$ & $n$ & $\%$ \\
\hline 1998 & 136 & 126 & 92.6 & 16 & 11.8 & 21 & 15.4 & 2 & 9.5 & 105 & 77.2 & 14 & 13.3 \\
\hline 1999 & 134 & 131 & 97.8 & 6 & 4.5 & 32 & 23.9 & 0 & 0.0 & 99 & 73.9 & 6 & 6.1 \\
\hline 2000 & 123 & 121 & 98.4 & 12 & 9.8 & 16 & 13.0 & 0 & 0.0 & 105 & 85.4 & 12 & 11.4 \\
\hline 2001 & 143 & 143 & 100.0 & 11 & 7.7 & 16 & 11.2 & 0 & 0.0 & 127 & 88.8 & 11 & 8.7 \\
\hline 2002 & 180 & 127 & 70.6 & 11 & 6.1 & 22 & 12.2 & 1 & 4.5 & 105 & 58.3 & 10 & 9.5 \\
\hline 2003 & 160 & 130 & 81.3 & 15 & 9.4 & 16 & 10.0 & 3 & 18.8 & 114 & 71.3 & 12 & 10.5 \\
\hline 2004 & 153 & 107 & 69.9 & 20 & 13.1 & 35 & 22.9 & 2 & 5.7 & 72 & 47.1 & 18 & 25.0 \\
\hline 2005 & 200 & 153 & 76.5 & 21 & 10.5 & 46 & 23.0 & 6 & 13.0 & 107 & 53.5 & 15 & 14.0 \\
\hline 2006 & 185 & 143 & 77.3 & 18 & 9.7 & 17 & 9.2 & 0 & 0.0 & 126 & 68.1 & 18 & 14.3 \\
\hline 2007 & 148 & 107 & 72.3 & 12 & 8.1 & 13 & 8.8 & 3 & 23.1 & 94 & 63.5 & 9 & 9.6 \\
\hline 2008 & 166 & 110 & 66.3 & 24 & 14.5 & 11 & 6.6 & 3 & 27.3 & 99 & 59.6 & 21 & 21.2 \\
\hline Sum & 1728 & 1398 & & 166 & & 245 & & 20 & & 1153 & & 146 & \\
\hline Mean & 157 & 127 & 82.1 & 15 & 9.5 & 22 & 14.2 & 2 & 9.3 & 105 & 67.9 & 13 & 13.1 \\
\hline
\end{tabular}

All all IR contributions, Prospective prospective contributions 
Fig. 3 Percentages of IR contributions for imaging modalities. $C T$ computed tomography, US ultrasound, MRI magnetic resonance imaging, IO-Non-CT ionising, non-CT imaging modalities

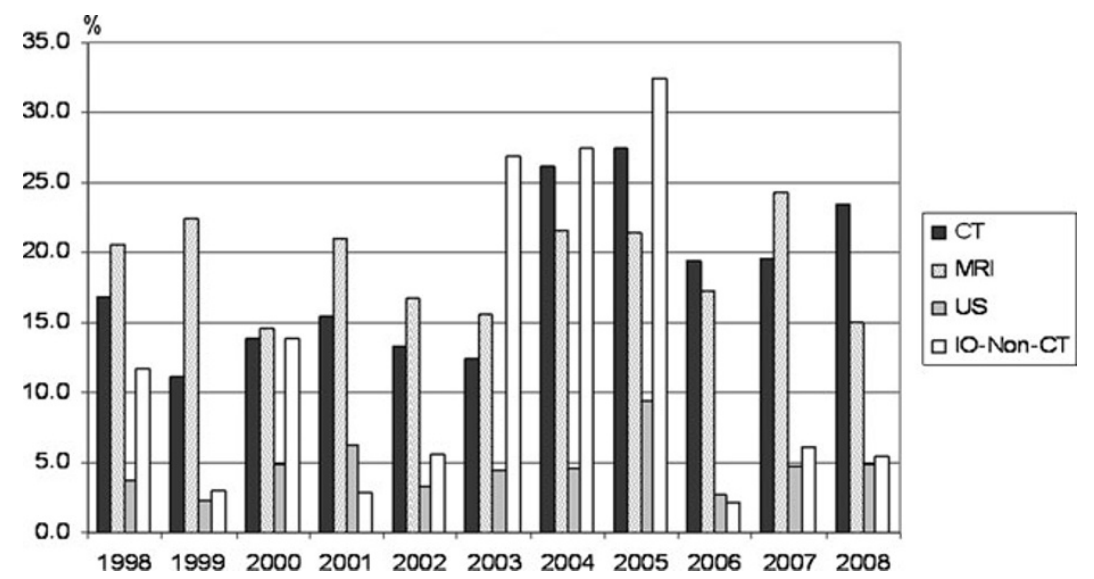

\section{Discussion}

IR has proven to be a stable and appreciated topic at the DRK - the largest congress of radiology in Germanspeaking countries and the third largest congress in Europe. With a proportion of IR abstracts between 15 and $20 \%$ of all contributions, IR rose significantly in absolute numbers to a peak of 200 abstracts in 2005 . This was primarily based upon structural changes of the DRK in the latter years clearly emphasising educational activities for radiologists and technicians and eliciting a substantial and significant growth of educational IR contributions. Given the increasing overall demand for educated staff in radiology, this is an impressive answer to persisting voices arguing for highlevel education to ensure the availability of properly trained radiologists [12-15]. In combination with the static time frame of the DRK, scientific IR contributions therefore declined only non-significantly. With $9-13 \%$ being prospective contributions, IR kept within an equal share of prospective scientific abstracts compared with general radiology [10]. The triplication of relative numbers for prospective contributions may be a calculation effect based on declining numbers of all scientific contributions and increasing numbers of prospective abstracts. Furthermore, absolute numbers of prospective contributions remainedunder postulation of evidence-based medicine as the goalquite small. This correlates to the warning comments of several authors about the amount and quality of IR literature and, moreover, demonstrates that despite requests for more research activities in IR [13, 16-18], only moderate increases of high-ranking studies have been reached in absolute numbers. Nevertheless, even when based on small absolute numbers, significantly more prospective research in IR has been presented at the DRK. The conflicting demands of raising absolute numbers of high-ranking prospective studies, managing increasing clinical workloads and-on the other hand-maintain the intensified educational activities, evidently advocate against further cutbacks in the equipment, manpower and funding of IR.

Image-guidance is without question an essential prerequisite of modern IR procedures. Given the clear advantages of CT imaging mentioned above, the rising demand of CT examinations in total and for CT-guided IR procedures is
Fig. 4 Percentages of IR contributions with respect to body regions. CNS central nervous system, including head/neck

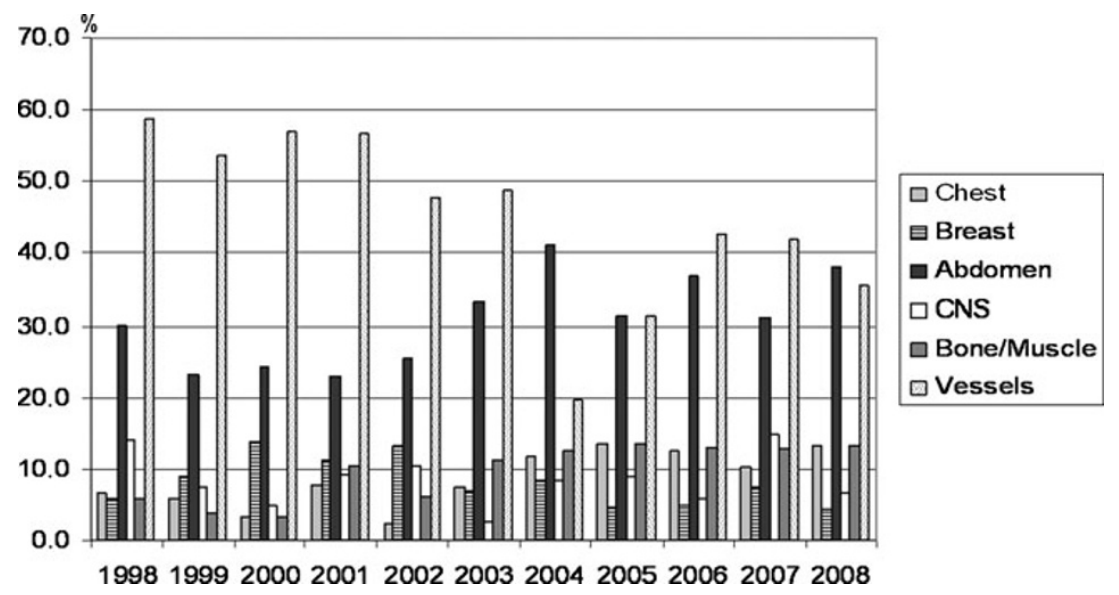


Fig. 5 Absolute numbers of IR abstracts relating to dose and all IR contributions (all IR all IR contributions at the DRK)

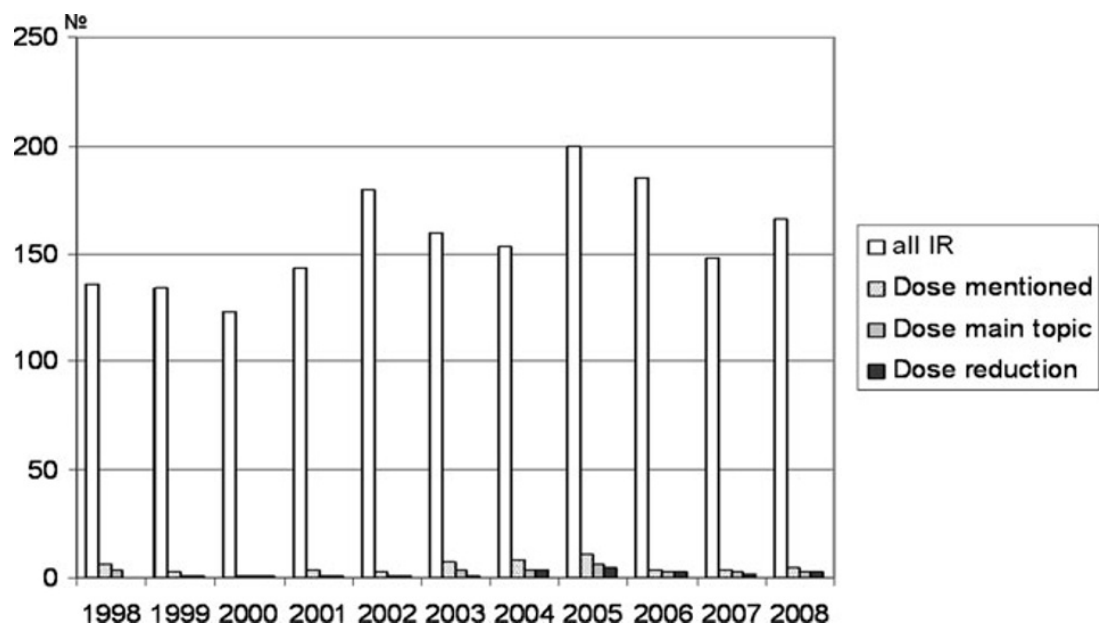

not surprising. Mettler et al. [19] reported in 2009 a tenfold increase in the number of all radiological procedures from 1950 to 2006 in the United States with a rise in the annual per-capita effective dose of $600 \%$. Citing a report of the National Council on Radiation Protection and Measurements, they presented an estimated number of 67 million CT examinations and 17 million interventional procedures in 2006 in the United States. With respect to IR, $72.2 \%$ of all interventional or catheterisation procedures in 2006 in the United States were non-cardiac diagnostic or therapeutic procedures applying $46.9 \%$ of the total collective dose with vascular, urinary and myelographic procedures being the most common areas of IR. Our results clearly show the significantly increasing use of CT with declining or stable proportions of MRI and other imaging modalities. The presented data indicate a shift of IR activities at the DRK from angiography-based vessel interventions to CT-guided procedures of the trunk: The traditional IR field of vessel interventions showed a drop in relative proportions with the main increment of IR being revealed within the regions of chest, abdomen, and the musculoskeletal system. These body regions mainly represent areas with the mostradiation-sensitive organs. In addition, the resulting effective doses of imaging procedures of the trunk - regardless of the chosen ionising imaging modality-are highest compared with all other body regions [20].

Based on the linear no-threshold model, low-dose diagnostic radiation exposure $(<100 \mathrm{mSv})$ accounts for a low but tangible risk of carcinogenesis [21-23]. Recent studies even report evidence for an increased risk for solid tumour development with exposures at a magnitude of 10 $50 \mathrm{mSv}$ [24-27]. Effective doses of fluoroscopic or angiographic IR procedures - especially when concerning the trunk as in embolisations or transjugular portosystemic shunt implantations - can be associated with a substantially increased likelihood of clinically significant patient doses [28]. As presented by Tsalafoutas et al. [29], median effective doses for CT-guided biopsies were about
Fig. 6 Percentages of IR abstracts relating to dose

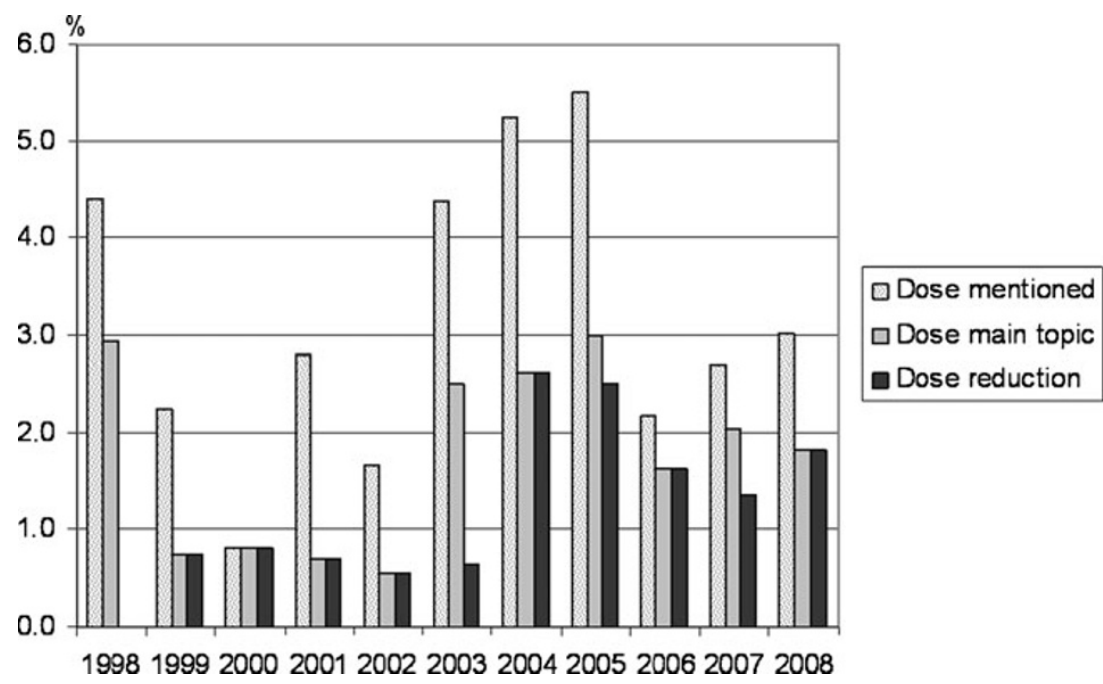




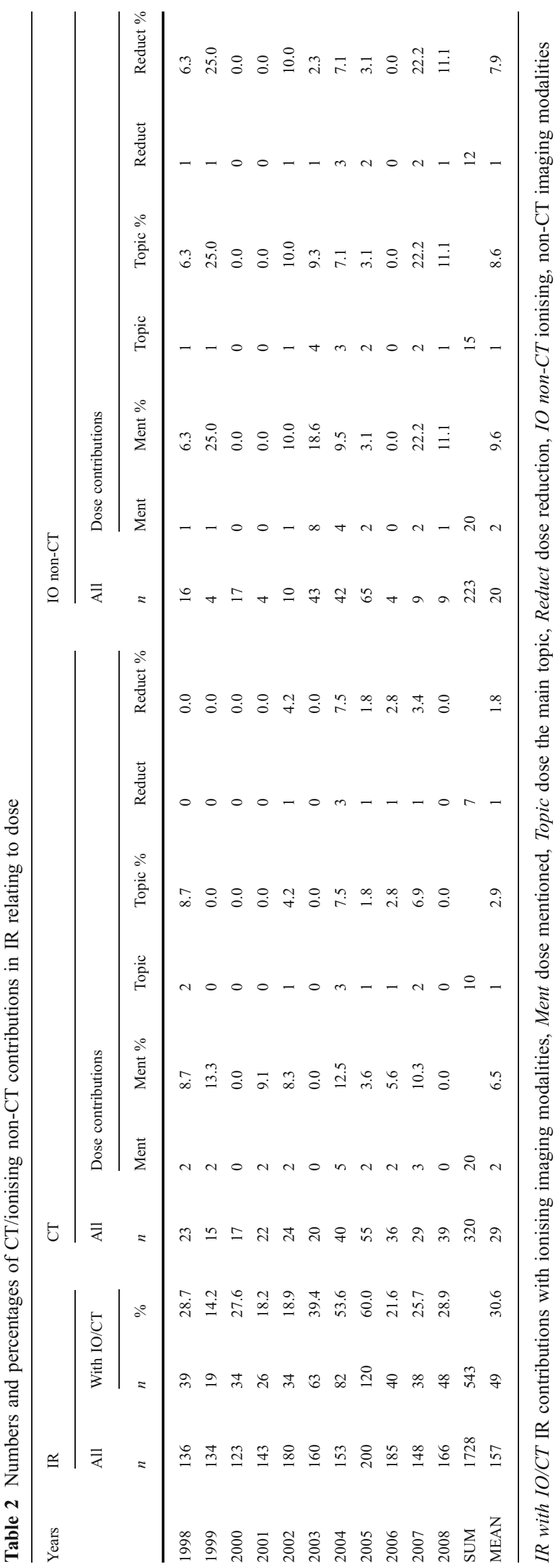

$23 \mathrm{mSv}$, for radiofrequency ablations $35 \mathrm{mSv}$, for abscess drainages $16 \mathrm{mSv}$ and for nephrostomies $11 \mathrm{mSv}$. The maximum effective dose of these procedures reached $57 \mathrm{mSv}$ and the unavoidable diagnostic part of the CTguided intervention produced the lion's share of radiation exposure. Although even these values of higher expositions are considered as "low" (i.e. one per 1,000 individuals) according to the proposed adequate risk terms of the UK Department of Health [30] compared with the general lifetime risk of cancer development, they are definitely above the effective doses accompanying most diagnostic procedures. Moreover, the increasing demand and sometimes repeated use of IR procedures make radiation protection an important issue. Studies revealed sobering results of the awareness and knowledge of non-radiologist healthcare professionals concerning the magnitude of radiation exposure combined with different radiological examinations, leaving a potential question mark on their ability to balance the risk-benefit ratio for a given patient [31-36]. Thus, interventional radiologists are by all means the primary correspondents of dose issues accompanying IR procedures. Despite small absolute numbers through the years for all dose categories, the presented results of our study demonstrate an increase in the awareness and occupation with radiation exposure measured in percentages of abstracts dealing with this topic at the DRK during the study period - concomitant with the above mentioned increase of IR abstracts in general. The significant increase of contributions in IR relating to dose reduction is especially encouraging. Obviously, the growing use of IR and ionising imaging modalities does not remain unanswered by interventionally acting radiologists with respect to radiation protection efforts. Nevertheless, compared with an analysis of general radiology and paediatric radiology in this 11-year period of the DRK presented elsewhere [9, 10], percentages of dose relevant contributions were lower in IR - especially for contributions with relation to CT. Furthermore, even when considering multiple naming for these dose categories, $94 \%$ of all IR abstracts at the DRK did not mention radiation exposure at all-despite the abovementioned increasing trend towards $\mathrm{CT}$ as the guiding instrument. Finally, looking at the absolute numbers of all IR contributions, of IR contributions pertaining to ionising procedures and for dose-related IR contributions (Table 2), the last category - even if growing — is clearly outnumbered.

Moreover, many abstracts did not report the target variable of radiation exposure-i.e. effective dose-for the mentioned procedures (neither expressed as typical doses for a given procedure nor based on their own calculated radiation data). This could have been for several reasons. First, effective dose as a calculated but not measurable variable represents in itself a concept that is combined with a relative uncertainty of up to $40 \%$ [30]. 
Some factors may influence the calculation of effective dose, especially factors as tissue weighting, scanning devices, region-based or organ-based calculation method and, finally, the patient's habitus compared with the proposed body model. Additionally, for some body regions tissue weighting factors are not disposable. Hence, some authors may have chosen to avoid the calculation of effective doses in favour of reporting measurable variables as the dose-length product orCT dose index. Moreover, the majority of evaluated abstracts concerned retrospective studies. It may have been impossible or to difficult to collect these data retrospectively - especially if radiation exposure was not in the focus of interest. Nevertheless, the seldom reporting of effective doses contradicts the intended use of this variable as a dose quantity that is easy to compare and - with respect to all its uncertainties - links the measured radiation dose to the risk of health detriment. Hence, it may be technically more precise to report the dose-length product or CT dose index, but the associated risk with a given procedure or for a given reference patient is not displayed. The contrast between this situation and the role of interventional radiologists as experts in radiation protection is potentially problematic. Moreover, it may be difficult to communicate to an incrementally attentive public, as issues dealt with at the DRK are frequently presented and discussed by the lay press. Given the interdisciplinary character of IR and the known turf battles around interventional medicine [1, 37, 38], deficiencies in the key qualification of radiation protection may ultimately heat discussions, which is why radiologists should be the primary experts and correspondents of IR. If radiologists want to maintain primacy in IR, further development of radiation protection knowledge based on intensified their own high-ranking research could be a cornerstone and unique selling proposition to succeed.

There are several limitations of this study, most important being the above-mentioned assumptions, upon which the evaluations were based. The first hypothesis, that the published abstracts contain all pertinent findings, does not exclude radiation exposure as a topic to be touched upon in the contribution but is not expressively mentioned in the abstract. Nevertheless, an author considering radiation exposure important enough to be discussed at the oral presentation, but not as eminent to be mentioned in the abstract, reflects an attitude towards the subject that is in itself problematic. Secondly, the DRK may not accurately reflect the current state of all scientific proceedings in Germany or other German-speaking countries. Moreover, as the number of accepted abstracts will have been different from the number of submitted abstracts to a given subject, there may have been several factors influencing the decision process of accepting contributions for the annual meetings of the DRK, including structural changes as mentioned above or the wish to even out contributions over different topics. But again, as the largest congress in the above-mentioned countries, there will be no better platform for such an analysis concerning the handling of ionising imaging modalities and dose in IR. Furthermore, the categorisation of the abstracts was intrinsically not immune to subjectivity, but over 11 years of annual meetings this would result in a systematic error probably factoring itself out. Moreover, because of the chosen study design, other indirect forms of radiation exposure reduction could have been missed. If, for instance, a study with comparison of an ionising and non-ionising imaging method in IR results in non-inferiority of the non-ionising imaging method, then this would also be a contribution to dose reduction, but would not have been included in our analysis. Finally, the question for the appropriate proportion of abstracts in a congress programme dealing with radiation exposure and dose reduction remains open.

In conclusion, IR emerged as a substantially growing specialty of radiology at the DRK from 1998 to 2008, with significant increments of educational activities and prospective research contributions. Despite a significant trend towards $\mathrm{CT}$ as an IR imaging modality, radiation exposure of IR was rarely in the focus of interest. Nevertheless, contributions relating to dose reduction demonstrated an encouraging and significant growth during the study period.

Acknowledgements Dr. Heyer has received honoraria for lectures from Bayer Vital, Bracco Altana, WAPPA, and from the scientific commission of the University Hospital "Bergmannsheil".

\section{Reference}

1. Lakhan SE, Kaplan A, Laird C, Leiter Y (2009) The interventionalism of medicine: interventional radiolgy, cardiology, and neuroradiology. Int Arch Med 2:27

2. Fioole B, van de Rest HJ, Meijer JR et al (2010) Percutaneous transluminal angioplasty and stenting as first-choice treatment in patients with chronic mesenteric ischemia. J Vasc Surg 51:386391

3. Stroszczynski C, Jores A, von Ruthendorf-Przewoski J, Bethke A (2008) Diagnostische punktion des thorax. Radiologe 48:11691172

4. Manchikanti L, Singh V, Pampati V, Smith HS, Hirsch JA (2009) Analysis of growth of interventional techniques in managing chronic pain in the medicare population: a 10 -year evaluation from 1997 to 2006. Pain Physician 12:9-34

5. O'Brien J, Baerlocher MO, Asch MR, Hayeems E, Kachura JR, Collingwood P (2007) Limitations influencing interventional radiology in Canada: results of a national survey by the Canadian Interventional Radiology Association (Cira). Cardiovasc Intervent Radiol 30:847-853

6. Manchikanti L, Pampati V, Singh V, Boswell MV, Smith HS, Hirsch JA (2010) Explosive growth of facet joint interventions in the Medicare population in the United States: a comparative 
evaluation of 1997, 2002, and 2006 data. BMC Health Serv Res 30:84

7. Kwan SW, Kerlan KRJ, Sunshine JR (2010) Utilization of interventional oncology treatments in the United States. J Vasc Interv Radiol 21(7):1054-1060

8. Ng KL, Yazer J, Abdolell M, Brown P (2010) National survey to identify subspecialties at risk for physician shortages in Canadian Academic Radiology Departments. Can Assoc Radiol J 61 (5):252-257

9. Heyer CM, Lemburg SP, Peters SA (2010) Paediatric radiology and scientific contributions to radiation dose at the meeting of the German Radiological Society - an analysis of an 11-year period. Eur J Radiol 75(2):e135-e140

10. Heyer CM, Peters S, Lemburg S (2009) Die Struktur des deutschen Röntgenkongresses und die wissenschaftliche Behandlung der Themen Strahlendosis und Dosisreduktion: Eine Analyse der Jahre 1998-2008. Rofo 181:1065-1072

11. Deutscher Röntgenkongress 1998-2008, Vollständiges Kongressprogramm und Abstracts der Wissenschaftlichen Beiträge. Thieme, Stuttgart New York

12. Amis ES Jr, Dunnick NR; American Board of Radiology, Diagnostic Radiology Residency Review Committee (2009) Improvement in Radiology Education: joint efforts of the American Board of Radiology and the Diagnostic Radiology Residency Review Committee. J Am Coll Radiol 6:103-105

13. Dodd GD, Fletcher TB, Thorwarth WT Jr (2006) The crisis in academic radiology: will we help ourselves? J Am Coll Radiol $3: 243-247$

14. Venkatesan AM, Shetty SK, Galdino GM, Davila JA, Lawrimore TM (2006) The impact of professional turf battles on radiology resident education: perspectives from the radiology class of 2005. J Am Coll Radiol 3:537-543

15. Vydareny KH (2004) The manpower crisis in academic radiology: don't kill the milk cow for meat. J Am Coll Radiol 1:100-103

16. Hillman BJ (2004) The need for clinical trials in radiology. J Am Coll Radiol 1:208-211

17. Levin DC, Rao VM (2007) Turf wars in radiology: the future of radiology depends on research - and on your support of it! J Am Coll Radiol 4:184-186

18. Medina LS, Blackmore CC (2007) Evidence-based radiology: review and dissemination. Radiology 244:331-336

19. Mettler FA Jr, Bhargavan M, Faulkner K et al (2009) Radiologic and nuclear medicine studies in the United States and worldwide: frequency, radiation dose, and comparison with other radiation sources - 1950-2007. Radiology 253:520-531

20. Lemburg SP, Peters SA, Roggenland D, Nicolas V, Heyer CM (2010) Cumulative effective doses of diagnostic imaging procedures in adolescents with spinal injuries. AJR Am J Roentgenol 195(6):1411-1417

21. Brody AS, Frush DP, Huda W, Brent RL (2007) Radiation risk to children from computed tomography. Pediatrics 120:677-682
22. Ron E (2003) Cancer risks from medical radiation. Health Phys $85: 47-59$

23. Sodickson A, Baeyens PF, Andriole KP et al (2009) Recurrent Ct, cumulative radiation exposure, and associated radiation-induced cancer risks from CT of adults. Radiology 251:175-184

24. Brenner DJ, Doll R, Goodhead DT et al (2003) Cancer risks attributable to low doses of ionising radiation: assessing what we really know. Proc Natl Acad Sci USA 100:13761-13766

25. Krestinina LY, Davis F, Ostroumova E et al (2007) Solid cancer incidence and low-dose-rate radiation exposures in the techa river cohort: 1956 2002. Int J Epidemiol 36:1038-1046

26. Pierce DA, Preston DL (2000) Radiation-related cancer risks at low doses among atomic bomb survivors. Radiat Res 154:178186

27. Preston DL, Shimizu Y, Pierce DA, Suyama A, Mabuchi K (2003) Studies of mortality of atomic bomb survivors. Report 13: solid cancer and noncancer disease mortality: 1950-1997. Radiat Res 160:381-407

28. Miller DL, Balter S, Cole PE et al (2003) Radiation doses in interventional radiology procedures: the Rad-Ir study: part I: overall measures of dose. J Vasc Intervent Radiol 14:711-727

29. Tsalafoutas IA, Tsapaki V, Triantopoulou C, Gorantonaki A, Papailiou J (2007) Ct-guided interventional procedures without Ct fluoroscopy assistance: patient effective dose and absorbed dose considerations. AJR Am J Roentgenol 188:1479-1484

30. Martin CJ (2007) Effective dose: how should it be applied to medical exposures? Br J Radiol 80:639-647

31. Heyer CM, Peters S, Lemburg S, Nicolas V (2007) Einschätzung der Strahlenbelastung radiologischer Thorax-Verfahren: was ist Nichtradiologen bekannt? Rofo 179:261-267

32. Lee CI, Haims AH, Monico EP, Brink JA, Forman HP (2004) Diagnostic Ct scans: assessment of patient, physician, and radiologist awareness of radiation dose and possible risks. Radiology 231:393-398

33. Quinn AD, Taylor CG, Sabharwal T, Sikdar T (1997) Radiation protection awareness in non-radiologists. Br J Radiol 70:102-106

34. Rice HE, Frush DP, Harker MJ, Farmer D, Waldhausen JH, Committee AE (2007) Peer assessment of pediatric surgeons for potential risks of radiation exposure from computed tomography scans. J Pediatr Surg 42:1157-1164

35. Shiralkar S, Rennie A, Snow M, Galland RB, Lewis MH, GowerThomas K (2003) Doctors' knowledge of radiation exposure: questionnaire study. BMJ 327:371-372

36. Thomas KE, Parnell-Parmley JE, Haidar S et al (2006) Assessment of radiation dose awareness among pediatricians. Pediatr Radiol 36:823-832

37. Levin DC, Rao VM, Bonn J (2005) Turf wars in radiology: the battle for peripheral vascular interventions. J Am Coll Radiol 2:68-71

38. Hobson RW 2nd, Howard VJ, Roubin GS et al (2004) Credentialing of surgeons as interventionalists for carotid artery stenting: experience from the lead-in phase of crest. J Vasc Surg 40:952-957 